\title{
Radiation-pressure-driven micro-mechanical oscillator
}

\author{
H. Rokhsari, T. J. Kippenberg, T. Carmon, and K. J. Vahala \\ Department of Applied Physics, California Institute of Technology, Pasadena, California, 91125, USA \\ vahala@caltech.edu \\ http://www.vahala.caltech.edu
}

\begin{abstract}
As Q factor is boosted in microscale optical resonant systems there will be a natural tendency for these systems to experience a radiationpressure induced instability. The instability is manifested as a regenerative oscillation (at radio frequencies) of the mechanical modes of the microcavity. The first observation of this radiation-pressure-induced instability is reported here. Embodied within a microscale, chip-based device reported here this mechanism can benefit both research into macroscale quantum mechanical phenomena [1] and improve the understanding of the mechanism within the context of LIGO [2]. It also suggests that new technologies are possible which will leverage the phenomenon within photonics.
\end{abstract}

(C)2005 Optical Society of America

OCIS codes: (140.4780) Optical resonators; (230.1040) Acousto-optical devices; (230.1150) All-optical devices.

\section{References and Links}

1. S. Mancini, V. Giovanetti, D. Vitali, and P. Tombesi, "Entangling macroscopic oscillators exploiting radiation pressure," Phys. Rev. Lett. 88, 120401 (2002).

2. V. B. Braginsky, S. E. Strigin, and S. P. Vyatchanin, "Parametric oscillatory instability in Fabry-Perot interferometer," Phys. Lett. A. 287, 331-338 (2001)

3. D. K. Armani, T. J. Kippenberg, S. M. Spillane, and K. J. Vahala K. J, "Ultra-high-Q toroid microcavity on a chip," Nature 421, 925-929 (2003).

4. V. B. Braginsky, I. I. Minakova, and P. M. Stepunin, "Absolute measurement of energy and power in optical spectrum according to electromagnetic pressure," Instrum. Exper. Tech-U. 3, 658-663 (1965).

5. V. B. Braginsky, and A. B. Manukin, "Ponderomotive effects of electromagnetic radiation," Sov. Phys. JETP-USSR. 25, 653-655 (1967).

6. A. Dorsel, J. D. Mccullen, P. Meystre, et al. "Optical bistability and mirror confinement induced by radiation pressure," Phys. Rev. Lett. 51, 1550-1553 (1983).

7. V. B. Braginsky, A. B. Manukin, and M. Y. Tikhonov, "Investigation of dissipative Ponderomotive effects of electromagnetic radiation," Sov. Phys. JETP-USSR. 31, 829-830 (1970).

8. The characteristics of the overall waveguide-resonator system can be viewed as an optical modulator that is driven by this oscillation. This modulator has a nonlinear transfer function that manifests itself (in the modulated pump power) through the appearance of harmonics of the characteristic mechanical eigenfrequencies. These harmonics are easily observed upon detection of the modulated pump (see Fig. 2).

9. For $f(d)<0$, i.e. a red shift of the pump frequency with respect to the cavity mode, the phase of the radiation pressure variations actually damps or "cools" the vibrations. Note that no external feedback system is necessary here to damp the vibrations or "cool" the resonator. The feedback system is inherent to the coupling mechanism. Due to the high quality factor of our cavities ( $~ \sim 10$ million) the "red shifted" tail of the optical mode is not thermally stable (see H. Rokhsari et. al. "Loss characterization in micro-cavities using the thermal bistability effect. Applied Physics Letters 85, 3029-3031 (2004)). Replacing the cavity material (silica) with a negative thermo-optic coefficient material would stabilize the red shifted tail and cavitycooling induced by radiation pressure effects could be observable.

10. M. Cai, O. Painter, and K. J. Vahala, "Observation of critical coupling in a Fiber taper to a silica microsphere whispering-gallery mode system," Phys. Rev. Lett. 85, 74-77 (2000).

11. For the sample tested, it is calculated that radial variations of about 10 picometers will shift the resonant frequency of the excited optical mode by its linewidth.

12. M. Zalalutdinov, et a, "Autoparametric optical drive for micromechanical oscillators," Appl. Phys. Lett. 79, 695-697. (2001).

13. C. H. Metzger, K. Karrai, "Cavity cooling of a microlever," Nature. 432, 1002-1005, (2004). 
14. T. J. Kippenberg, H. Rokhsari, T. Carmon, and K. J. Vahala, accepted by Phys. Rev. Lett.

15. H. Rokhsari, .S. M. Spillane, and K. J. Vahala, "Observation of Kerr nonlinearity in microcavities at room temperature," Opt. Letts. 30, 427-429 (2005).

16. V. S. Ilchenko, and M. L. Gorodetsky, "Thermal nonlinear effects in optical whispering gallery microresonators," Laser Phys. 2, 1004-1009 (1992).

17. We note that as evident in the renderings provided in Figs. 1 and 2, the $n=3$ mechanical mode has a strong radial component to its motion and hence understanding of its excitation by way of radiation pressure (which itself is primarily radial in direction) is straightforward. In contrast, the $n=1$ mode motion is transverse, requiring a different method of force transduction. The details here, including threshold calculations, will be presented in a forthcoming article where it is shown that minute offsets of the optical mode from the equatorial plane provide a moment arm for action of radiation pressure. The resulting torque induces the transverse motion associated with the $n=1$ mode. Modelling, including an SEM measurement of the offset, confirms this mechanism.

18. X. M. H. Huang, C. A. Zorman, M. Mehregany, and M. L. Roukes, "Nanodevice motion at microwave frequencies," Nature. 421, 496 (2003).

19. W. Kells, and E. D'Ambrosio, "Considerations on parametric instability in Fabry-Perot interferometer," Phys. Lett. A. 299, 326-330 (2002).

20. V. B. Braginsky, S. E. Strigin, and S. P. Vyatchanin, "Analysis of parametric oscillatory instability in power recycled LIGO interferometer," Phys Lett. A. 305, 111-124 (2002).

21. S. W. Schediwy, C. Zhao, L. Ju, et al, "An experiment to investigate optical spring parametric instability," Classica Quant. Grav. 21, S1253-S12587 (2004).

22. I. Tittonen, et al, "Interferometric measurements of the position of a macroscopic body: Towards observation of quantum limits," Phys. Rev. A. 59, 1038 (1999);

23. B. Julsgaard, A. Kozhekin, E. S. Polzik, "Experimental long-lived entanglement of two macroscopic objects," Nature (London), 413, 400 (2001).

24. V. Giovanetti, S. Mancini, P. Tombesi, "Radiation pressure induced Einstein-Podolsky_Rosen paradox," Europhys. Lett. 54, 559-565, (2001).

25. S. Pirandola, S. Mancini, D. Vitali, and P. Tombesi, "Continuous-variable entanglemet and quantum-state teleportation between optical and macroscopic vibrational modes through radiation pressure," Phys. Rev. A., 68, 062317, (2003); W. Marshall, C. Simon, R. Penrose, and D. Bouwmeester, "Towards Quantum

Superpositions of a Mirror," Phys.Rev. Lett. 91, 130401, SEP (2003).

In this work, the radiation-pressure instability [2] is observed for the first time. The coupling of optical and mechanical degrees-of-freedom caused by radiation pressure in a microcavity is shown to produce regenerative oscillation of the cavity mechanical degrees of freedom. The oscillations are observed here in a whispering gallery toroid microcavity [3]. These devices, which exhibit ultra-high-Q factors, provide but one possible platform on which the oscillations can be observed; in particular, as Q factor rises in other microcavity systems, the tendency towards this same effect should become apparent.

Microtoroid structures are described elsewhere [3], however, in brief, they are made of silica and attached to a silicon cylindrical pillar (see Fig. 1). When coupled to a waveguide, the high quality factor of micro-toroids $\left(\sim 10^{8}\right)$ results in optical power build-up that can exceed 100 Watts of circulating power for only $1 \mathrm{mWatt}$ of waveguide input power. The resulting radiation pressure [4-7] due to confinement of these high optical powers in micron-scale volumes excites the vibrational modes of the toroidal structures. At an intuitive level, this excitation process can be understood as follows. Assuming first that the optical pump wave frequency $(\omega)$ is nearly resonant (but not exactly resonant) with the optical mode, radiation pressure, caused by circulating photons inside the cavity, will induce deformation of the toroidal structure and change the resonant frequency of the micro-cavity through dimensional changes in the modal round-trip optical path length. This resonant shift either lowers or raises the coupled optical pump power, depending upon the sign of the detuning of pump frequency relative to the microcavity resonant frequency. It will be shown that when the pump laser is detuned to the high-frequency tail of the optical mode, the phase relationship between optical pressure and microcavity deformation results in net power transfer from the optical pump to the mechanical mode. This transfer manifests itself mathematically as a mechanical gain, for the mechanical oscillations, with a corresponding threshold optical power. Interaction of the vibrating resonator (at frequency $\Omega$ ) with photons inside the cavity results in creation of photons down-shifted (stokes sideband, $\omega-\Omega$ ) or up-shifted (anti-stokes sideband, $\omega+\Omega$ ) 
in energy from the original photons by the RF frequency of the vibrations. Beating of the original pump wave with the up/down-shifted, optical sidebands therefore results in oscillations in optical power transmission at the mechanical frequencies of the structure. A rendering of this process is provided in Fig. 1.
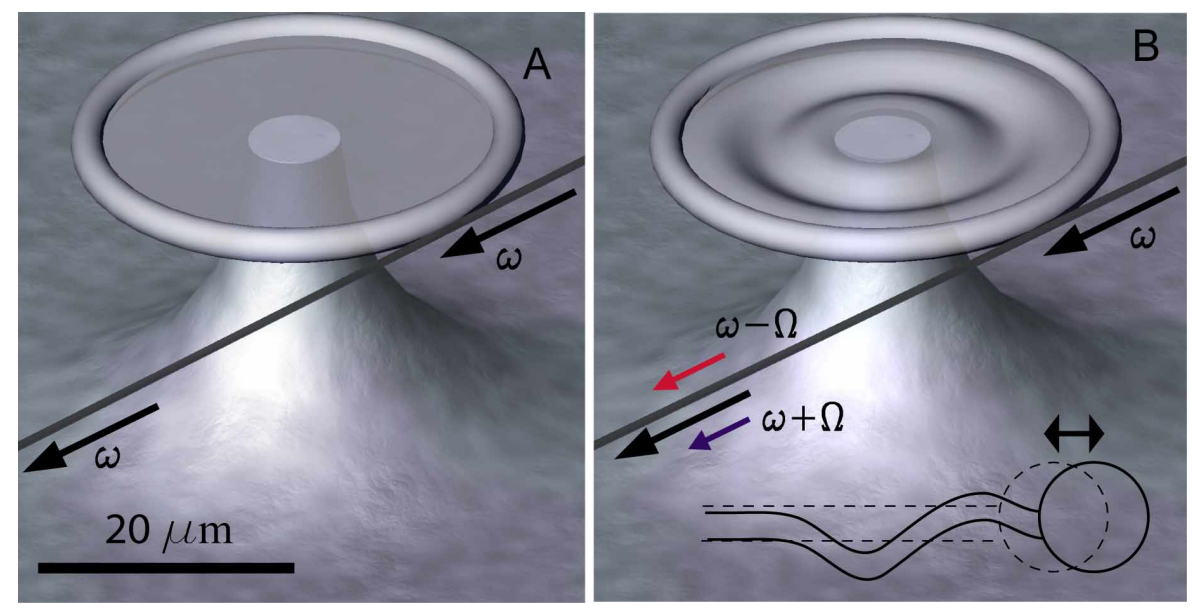

Fig. 1. Panel A illustrates the "below threshold" behavior where the optical pump wave at frequency $\omega$ is not strong enough to induce mechanical oscillations of the micro-toroid. Panel B illustrates the "above threshold" case for the $n=3$ vibrational mode. Mechanical oscillation at frequency $\Omega$ creates optical stokes $(\omega-\Omega)$ and anti-stoked sidebands $(a+\Omega)$ in the transmitted pump wave. Inset of panel B shows the exaggerated cross-section of the third order mode and variation of the toriod radius as a result of these oscillations.

We observed these oscillations when the optical pump is coupled to sufficiently high quality factor optical modes ( $Q \approx 10^{7}$ or $10 \mathrm{~ns}$ photon lifetime at infra-red wavelengths). Spectral analysis of the detected, transmitted optical power using a high-resolution electrical spectrum analyzer (see Fig. 2) revealed extremely narrow peaks (linewidths of less than $10 \mathrm{~Hz}$ limited by the equipment resolution) at a frequency typically in the range of $10-100 \mathrm{MHz}$ as well as at harmonics of this fundamental frequency. As can be seen in Fig. 2, two, distinct fundamental oscillation frequencies (and their harmonics [8]) were observed: a low frequency mode $(\sim 2-20 \mathrm{MHz})$ and a high frequency mode $(\sim 40-100 \mathrm{MHz})$. 


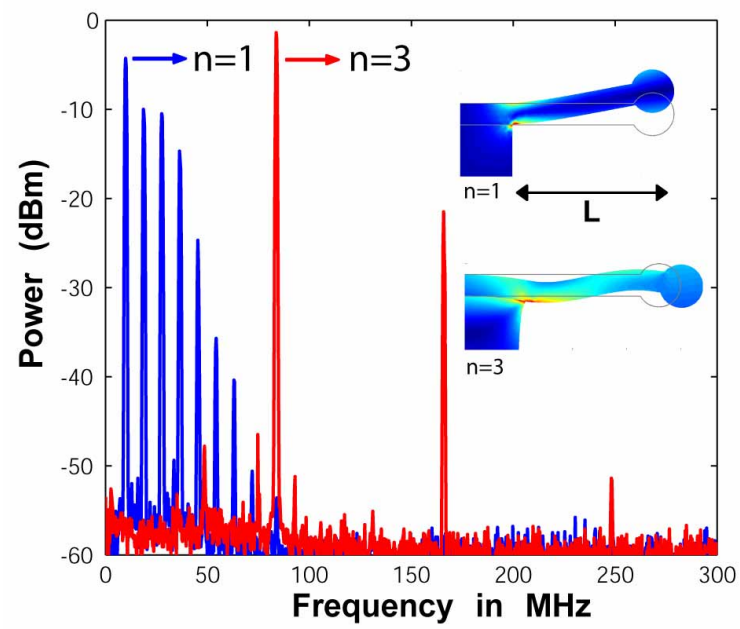

Fig. 2. The measured, spectral content of pump-power (at $1550 \mathrm{~nm}$ ) transmission as observed on an electrical spectrum analyzer (bandwidth set at $100 \mathrm{kHz}$ ). Two families of frequencies are observed. Those at lower frequency are driven by oscillation of an $n=1$ vibrational mode and those at higher frequency by an $n=3$ mode. Harmonics of the fundamental mechanical frequency are caused by the nonlinear transfer characteristic of the waveguide resonator system (see footnote 8 ). The inset shows the numerically modelled cross-sectional plot (exaggerated for clarity) of the strain field for the first and third vibrational eigen-modes of a toroidal silica micro-cavity on a silicon post. The stress field is superimposed (color coded). As evident from the modelling, the mechanical oscillations cause a displacement of the toroidal periphery and thereby induce a shift in the whispering gallery mode resonant frequency.

The eigen-frequencies of the mechanical modes of the toroidal structures were investigated numerically to confirm their mechanical origin. Close agreement of the measured RF oscillation frequencies with the results of the numerical modelling (less than $2 \%$ discrepancy) confirms that the first $(n=1)$ and third $(n=3)$ order flexural modes are responsible for creating the observed low- and high- frequency families of oscillations, respectively. The inset in Fig. 2 shows the numerically modelled cross-sectional view of the $n=1$ and $n=3$ modes, while Fig. 3 provides the comparison between the numerical and experimental mechanical eigenfrequencies versus the toroid "overhang length" L (see cross sectional view in Fig. 2 for the definition of L). The mechanical origin of these oscillations was also confirmed by lowering a metallic micro-probe into proximity with the plane of the silica disk connecting the toroid to the silicon pillar (i.e. not the toroid itself, where the optical mode lies). Since the probe is far removed from the toroidal whispering gallery, it affects only the mechanical and not the optical properties of the structure. The optical power oscillations were observed to fully quench upon probe contact. 


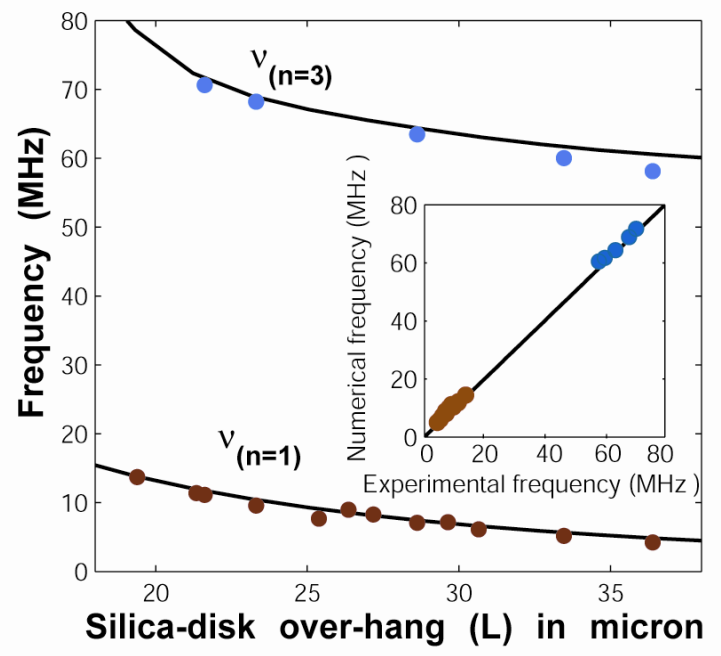

Fig. 3. Numerical calculation (solid line) and measured (points) dispersion relation for the fundamental $(n=1)$ and third order $(n=3)$ flexural modes of a micro-toroid as a function of the free hanging length of the disk structure. The inset shows the agreement between the numerical and measured frequencies.

This system can be modelled using a set of coupled differential equations: one governing the harmonic motion of the flexing toroid and a second governing the resonant optical field [2]. The equation of motion for deformation (more precisely, the displacement of the whispering gallery) is that of a damped, harmonic oscillator driven by radiation pressure (generated by the circulating optical field at the periphery of the micro-toroid). The optical field in the cavity, on the other hand, couples to this mechanical motion through the dependence of the cavity resonant frequency on mechanical deformation. By solving this system of equations selfconsistently a mechanical gain term can be defined which is a linear function of the circulating optical power and that offsets intrinsic sources of mechanical damping as given below. This leads to a threshold optical pump power for onset of mechanical oscillations as follows:

$$
\gamma=\gamma_{0}\left(1-P / P_{\text {threshold }}\right), \quad P_{\text {threshold }}=\mathrm{K}_{\text {opt-mech }} \frac{Q_{c}}{Q_{\text {total }}^{4} Q_{\text {mech }}} f(d)
$$

where the intrinsic mechanical damping coefficient $\gamma_{0}$ is modified to $\gamma$ in the presence of the optical power in the waveguide $\left(P\right.$ ) and where $P_{\text {threshold }}$ denotes the incident (in the waveguide) threshold power (i.e., not the power coupled into the resonator). Also, $Q_{\text {total }}$ is the total quality factor of the optical mode, (made up of an intrinsic contribution $Q_{0}$ and a waveguide-loading contribution $\left.Q_{c}: 1 / Q_{\text {total }}=1 / Q_{0}+1 / Q_{c}\right)$, and $Q_{\text {mech }}\left(=\Omega / \gamma_{0}\right)$ is the intrinsic quality factor of the mechanical mode. $d$ is the detuning factor, giving the normalized detuning of the optical frequency from the resonant optical frequency of the cavity $\left(\omega_{0}\right)$ in units of its linewidth (i.e. $\left.d \equiv Q_{\text {total }} \frac{\omega-\omega_{0}}{2 \omega_{0}}\right)$ and $f(d)=\left(1+d^{2}\right)^{3} / d$ is a factor that is positive for blue-detuning of the pump (and negative for red detuning). This factor, emerging from the analysis, reflects the intuitive picture described above requiring a particular phase relation between the variation in coupled radiation pressure and cavity deformation in order that power is transferred from the optical mode to the mechanical mode [9]. The 
constant $\mathrm{K}_{\text {opt-mech }}=R^{2} \omega_{0}^{2} m \Omega / 64$, determines how strongly the optical and mechanical modes are coupled and depends upon the radius $R$ of the micro-toroid, its vibrational frequency $\Omega$ and the vibrating mass $m$. Equation (1) shows that the threshold power scales approximately with $1 / Q_{\text {total }}^{3}$, and emphasizes the importance of high optical Q.

In order to verify the predictions of equations (1), the mechanical damping rate was measured as a function of optical pump power in the sub-threshold regime. In this regime, damping of mechanical oscillations can be decreased by injecting optical power into the microtoroid, yet the induced mechanical gain is not sufficient to initiate the regenerative vibrations. To measure the damping rate, an optical pump and probe approach was used with two laser beams (a strong pump and a weak probe) individually resonant with two whispering gallery mode optical resonances. Two external cavity diode lasers with $300 \mathrm{kHz}$ linewidth were used as laser sources. The pump laser in the $1550 \mathrm{~nm}$ band and probe laser in the $1480 \mathrm{~nm}$ band were coupled to the micro-cavity using a tapered optical fiber formed by heating and stretching a length of single mode fiber [10]. The output of the $1550 \mathrm{~nm}$ laser was weakly modulated using a Mach-Zehnder modulator. In this way the "DC" component of the pump power creates gain for the mechanical modes while the alternating component creates a harmonically-varying radiation-pressure which acts as a forcing function on the sub-threshold vibrational modes. Since the response motion causes frequency shifts of the optical mode that is resonant with the probe signal, the mechanical response as a function of forcing-function frequency can be measured using the optical probe wave. The resulting modulation of the probe power can be measured using a lock-in amplifier and it can be related to the amplitude of vibrations by measuring the quality factor of the optical mode coupled to the probe wave and its detuning from probe signal (for these experiments the weak probe beam was coupled to a low Q $\left(\sim 10^{6}\right)$ whispering gallery resonance to ensure that probe power would not induce a competing oscillation effect). In this way, "vibrational spectroscopy" can be performed by sweeping the modulation frequency through the vibrational resonances. This spectroscopy reveals the lineshape of the mechanical resonance and hence its linewidth (and damping rate or equivalently the mechanical $\mathrm{Q}$ factor). Figure 4 gives sample spectroscopic scans over an $\mathrm{n}=1$ vibrational mode with a resonant frequency of $7.67 \mathrm{MHz}$. The solid lines are the theoretical fits using a damped harmonic oscillator model for the mechanical motion. The inset in Fig. 4 gives measured damping rates (extracted from the theoretical fits like that shown in the main Fig.) plotted versus optical pump power using the pump/probe spectroscopy measurements. A linear decrease in damping with increasing pump power is evident in the data (in agreement with equation 1). Also apparent is the threshold power, which in this case is expected to occur at an optical pump power of 11 micro-Watts. The intrinsic $\mathrm{Q}$ factor of the mechanical mode (i.e., $\mathrm{Q}$ factor at zero input pump power) is inferred to be 630 in this data. We have observed intrinsic mechanical quality factors in excess of 5,000 in toroidal structures.

$\# 7413$ - \$15.00 USD

(C) 2005 OSA
Received 10 May 2005; revised 23 June 2005; accepted 24 June 2005 11 July 2005 / Vol. 13, No. 14 / OPTICS EXPRESS 5298 


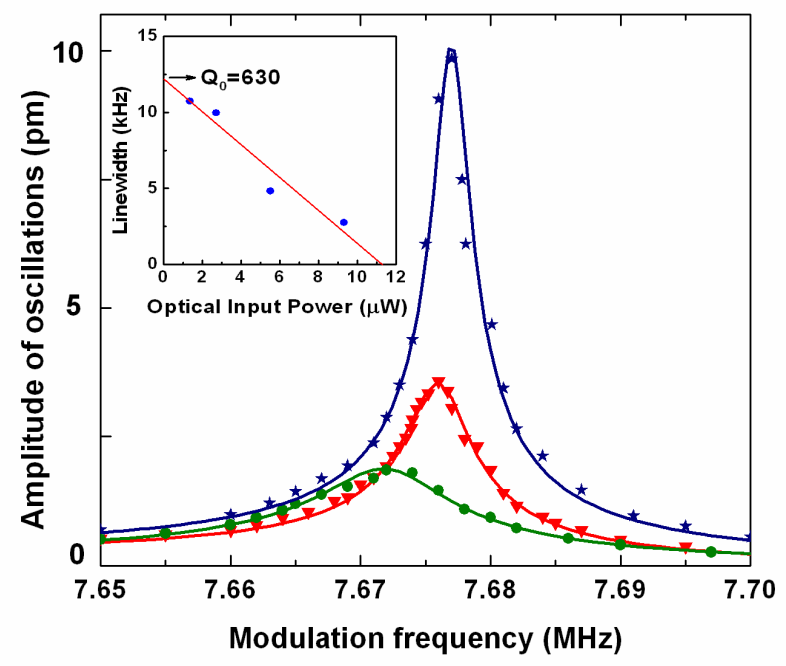

Fig. 4. Measured amplitude response (points) of the mechanical vibrations of an $n=1$ mechanical mode as a function of driving-force frequency (modulation frequency of the pump power). Circles (green), triangles (red), and stars (blue) represent the data for $2 \mu W, 5 \mu W$ and $9 \mu W$ of average pump power. The inset shows the effect of the optical power on the linewidth of the mechanical oscillator inferred from the theoretical fits (such as the solid lines in the main Fig.). A linear fit shows a threshold of $11 \mu W$ for the mechanical oscillations and an intrinsic quality

factor of 630 for the measured mechanical mode of the toroidal structure.

To characterize the mechanical modes in the above-threshold regime, the weak modulation of the pump power was terminated (i.e., steady pump wave) and the spectral content of the transmitted probe power was analyzed to monitor regenerative mechanical oscillation. The Fourier component of the transmitted probe power at the mechanical resonant frequency $(\Omega$ ) was monitored by the electrical spectrum analyzer. The intensity of this signal, proportional to the amplitude of the vibrations caused by the pump wave, was measured as a function of pump power. Figure 5 contains a typical result of these measurements for one of the studied devices and shows a clear threshold for the vibrational oscillations. Since the oscillator behaves as an optical power modulator [8] the electrical spectrum analyzer data can be transformed to compute the actual amplitude of mechanical motion. In other words modulation depth in the optical power knowing the linewidth of the optical mode can be transformed into frequency variation caused by the mechanical motion. This frequency variation however is directly proportional to the ratio of amplitude of the mechanical motion to the radius of the microtoroid. This transformation has been used to calibrate the vertical axis in Fig. 5. The data in Fig. 5 also seem to suggest that the amplitude of the vibrations saturates at high pump powers. Numerical modelling to be provided elsewhere shows that this behaviour can be attributed to the induced frequency shifts of the cavity which, for higher pump power levels, exceed the cavity linewidth. This, in turn, reduces the efficacy of the pumping mechanism as the pump wave spends a progressively smaller fraction of time on resonance during each mechanical cycle [11]. 


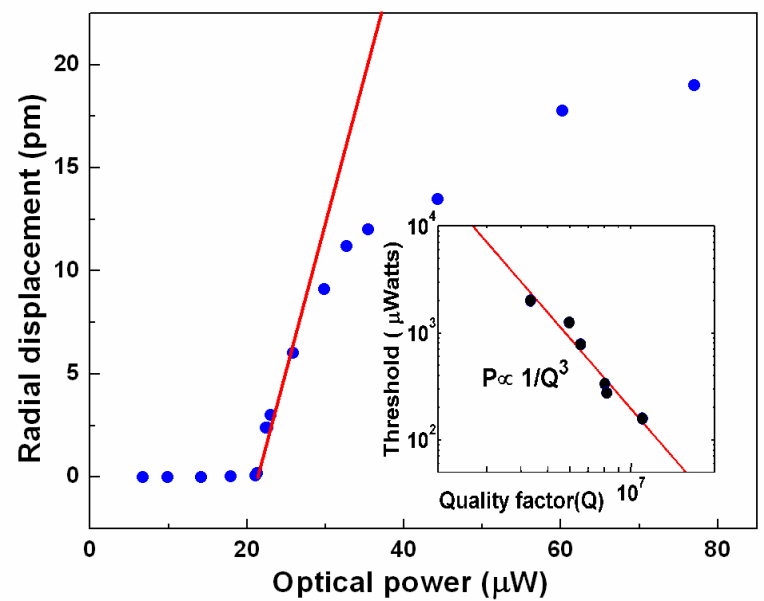

Fig. 5. Measured mechanical oscillator displacement as a function of the optical pump power showing threshold behaviour. Oscillations initiate at about $20 \mu W$ of input power and start to saturate for higher values of pump power. This saturation is associated with the lower opticalmechanical coupling at displacements large enough to shift the resonant frequency of the optical mode by greater than its linewidth. Inset shows the dependence of measured threshold power on optical quality factor. Data taken from Ref. [14]. These data were obtained using different optical modes in a single micro-toroid (so that only optical Q would be varied). The slope of the fit in a log-log scale is $3 \pm 0.3$, which agrees well with inverse cubic behaviour expected for radiation-pressure-induced regenerative oscillations (as opposed to inverse quadratic behaviour for photothermal effects).

Mechanical oscillations in micro-structures can be generated using alternative methods. For example, Ref. 12 and Ref. 13 describe thermally actuated mechanical vibrations of a silicon disk and a silicon cantilever respectively. In contrast, the long thermal time constant of the toroid structures in the present work ( $\tau_{\text {thermal }}$ is in the order of $5 \mu s[15,16]$ ) precludes initiation of RF rate ocsillations such as those observed here. Also significant is the expected threshold dependence on optical Q for thermally driven instabilities. Because resonator deformation for a thermally driven process (as opposed to radiation pressure) depends on coupled optical power (not circulating power) one expects an inverse quadratic scaling of the threshold power with optical Q $\left(P_{\text {threshold }} \propto Q^{-2}\right)$ for thermally-induced oscillations as opposed to inverse cubic ( $P_{\text {threshold }} \propto Q^{-3}$ ) for radiation pressure induced oscillations. This $\mathrm{Q}$ dependence has been verified in the inset of Fig. 4, further confirming radiation pressure as the origin of the observed mechanical oscillations. For these measurements, the threshold power was measured using many different optical modes (having differing optical Qs) within the same microtoroid device. In this way, only optical Q was varied. In addition, direct comparison of the measured threshold power with predictions of equation (1) results in typically less than $20 \%$ discrepancy further confirming the predictions of the model. As an example, one of the samples with $Q_{\text {total }}=2 \times 10^{6}$ and $d \approx 1$ exhibited threshold power of $520 \mu \mathrm{W}$ for the $\mathrm{n}=3$ mode [17] at $\Omega=42.3 \mathrm{MHz}$. The resonator had a major radius of about $23 \mu \mathrm{m}$, a mechanical Q factor of $Q_{m}=210$, and mass of $\left(m \approx 3 \times 10^{-11} \mathrm{Kg}\right.$ ). Using these parameters in equation (1) results in a theoretical threshold estimate of $500 \mu \mathrm{W}$, which is within $4 \%$ of the measured value. Note that this calculation includes no free parameters. 
The above findings demonstrate a new class of hybrid oscillator where a continuous source of pump laser power generates radio frequency mechanical vibrations of a micro-scale structure (without utilizing any type of external feedback system). These oscillations imprint onto the optical pump, now an optical carrier of RF frequencies. Realization of this effect is by no means restricted to the toroidal micro-cavities as we were also able to observe similar oscillations in silica micro-spheres. Therefore it is likely that all optical cavities are susceptible to these oscillations at various optical powers. The inverse cubic dependence of threshold power suggests that current efforts directed towards realization of higher $\mathrm{Q}$ optical microcavities will only tend to accelerate the observation of these oscillations in other microcavity systems. Beyond applications in RF micro-mechanical oscillators [18] on a chip, the ramifications of the radiation pressure induced opto-mechanical coupling have been theoretically explored in a variety of fundamental studies. In the Laser Interferometer Gravitational Wave Observatory (LIGO) community, it is proposed during the past few years $[2,19,20]$ that the so-called parametric instability could potentially limit the maximum stored energy in Fabry-Perot cavities used in the LIGO project and hence also the sensitivity of the gravitational wave detector. Recently, a bench top experimental setup was proposed to verify these theoretical concerns on a smaller scale [21]. This effect, never before observed, is more likely to occur in optical micro-resonators, as the threshold power scales rapidly with

dimensions (see equation 1 and definition of $\mathrm{K}_{\text {opt-mech }}$ ). The work presented here is to the authors' knowledge the first demonstration of the radiation pressure induced parametric instability in optical resonators of any kind. Cavity cooling experiments [13], standard quantum limited measurements of position [22], and the entanglement of light and macroscopic objects [23] are other exciting areas where this interaction can become useful. For example radiation pressure acting on a movable cavity can transfer information between two systems initially in semiclassical states, which, in turn, become entangled [24]. Using this entanglement it is possible to perform Einstein-Podolsky-Rosen (EPR) tests. Also, the possibility of teleporting and storing the quantum information carried by the radiation field in the vibrational state of a macroscopic cavity through ponderomotive entanglement, proposed recently, $[1,25]$ could benefit from the present work.

\section{Acknowledgments}

This work was supported by DARPA, NSF and the Caltech Lee Center. H.R. acknowledges Denis Armani for his generous help and T.J.K. acknowledges a postdoctoral fellowship from the CPI.

$\# 7413$ - \$15.00 USD

(C) 2005 OSA
Received 10 May 2005; revised 23 June 2005; accepted 24 June 2005 11 July 2005 / Vol. 13, No. 14 / OPTICS EXPRESS 5301 